\title{
Research of Methods of Theory and Application about Moving Object Detection
}

\author{
Yong Qin ${ }^{1,2}$, Junhua Han ${ }^{1,2}$, Meng Wei ${ }^{1,2}$, Hao Luo ${ }^{1,2}$ and Wenting Fan ${ }^{3}$ \\ ${ }^{1}$ College of Measure-Control Technology and Communication Engineering, \\ Harbin University of Science and Technology, \\ HeiLongJiang Harbin 150080, China \\ ${ }^{2}$ the Higher Educational Key Laboratory on Measuring \& Control \\ Technology and Instrumentations of Heilongjiang Province, \\ HeiLongJiang Harbin 150080, China \\ ${ }^{3}$ School of Electrical Engineering and Automation, \\ Harbin Institute of Technology, China 150080 \\ longmenmail@163.com,hjh198773@126.com
}

\begin{abstract}
In view of the shortcoming and the insufficiency of the traditional video monitoring system is complex, the power and the cost is higher, cost a lot of human resources. This paper design a moving objects detection system on the basis of the traditional video monitoring system in moving target detection algorithm, which applied to special environment conditions. The design in this paper uses ARM processor as the hardware platform and embedded operation system as software platform, uses the algorithm combined the background subtraction method and the frame difference, realizes fast image recognition of moving objects. This system alarms, saves the moving object images, and sends the images to the monitor by mail. The experiment simulation results show that the system detects moving objects fast, accurate, stable, good real-time performance, so it can be applied to unmanned environment monitoring etc and has good market prospects.
\end{abstract}

Keywords: moving target detection, background subtraction method, frame difference, unmanned environment

\section{Introduction}

In recent years, with the development progress of society, life rhythm of people speeds up. Unmanned environment often appears in the family and the work environment [1]. At this time, there are serious security problems, and a security video monitoring system is necessary. But the traditional video monitoring has two major defects [2]. One is that the monitor has to monitor the activities of the scene. If the monitor continuous monitoring for a long time, his attention will fall and not easy to find the anomalies in the scene. The other is when the anomalies appear in the monitoring scene, the monitor has to query the entire monitoring video in chronological order. This needs to spend a lot of time and energy. Aiming at unmanned indoor environment, this paper designs a kind of moving target detection system, recognizes the moving objects in the static fast and alarms.

In video monitoring system, moving target detection algorithm is the important manifestation of its intelligent level [3]. Many experts and scholars at home and abroad have a series of breakthrough achievements on the motion detection algorithm. But because the 
influence of the speed of moving object, light in the environment, the complexity of the algorithm etc., it is difficult to find a kind of effective algorithm to accurately complete extract moving object[4]. This design combines the background subtraction method and the frame difference, makes the two algorithms complementary advantages, overcomes their own weaknesses, realizes the detection of moving objects fast and exactly and further improves the stability of the monitoring system.

This design not only helps to solve the traditional monitor shortcomings, improves the stability and safety of the monitoring system, but also has more practical significance. And this design is applied in the unmanned environment of life work, the base station monitoring of telecommunications industry, the night monitoring of the museum and so on.

\section{Moving Object Intrusion Detection Algorithm}

Many domestic and international scholars have an in-depth study on moving object detection algorithm. Now popular moving object detection algorithm mainly has been divided into three kinds, optical flow, frame difference, background subtraction method $[5,6]$.

The principle of optical flow is mainly to detect the characteristics of moving objects along with the time changed. Optical flow is mainly suitable for camera constant motion of the moving target detection, but due to the high complexity of the algorithm, higher cost of hardware, and the computer time-consuming takes longer and can't meet the real-time.

The principle of frame difference is comparing the image sequence of two or three consecutive frames corresponding pixel gray level difference to the threshold to extract the image motion in the area [7]. This algorithm is simple, with rapid detection speed and good real-time, but influenced by the speed of the moving object. To the slower moving objects the detection information is incomplete.

The formula of frame difference is

$$
f_{j}(x, y, \mathrm{t})=\left|f_{m}(\mathrm{x}, \mathrm{y}, \mathrm{t})-f_{n}(\mathrm{x}, \mathrm{y}, \mathrm{t}-1)\right|
$$

Where $f_{m}(\mathrm{x}, \mathrm{y}, \mathrm{t})$ is the input image of current frame, $f_{n}(\mathrm{x}, \mathrm{y}, \mathrm{t}-1)$ is the previous frame image, $f_{j}(x, y, \mathrm{t})$ is the adjacent frame difference image.

For moving objects detection is based on the computation of the threshold $\mathrm{X}$, through judging whether adjacent frame difference image is greater than the threshold to judge whether the image is hacked by objects.

The principle of background subtraction method subtracts the current input image from the background model, so as to achieve to detect the target area of moving object [8]. Its advantage is small amount of computation, and can quickly complete to detect intrusion objects. But this algorithm has higher requirements for the light.

The formula of background subtraction method is

$$
f_{j}(x, y, \mathrm{t})=\left|f_{m}(\mathrm{x}, \mathrm{y}, \mathrm{t})-f_{n}(\mathrm{x}, \mathrm{y})\right|
$$

Where $f_{m}(\mathrm{x}, \mathrm{y}, \mathrm{t})$ is the input image of current frame, $f_{n}(\mathrm{x}, \mathrm{y})$ is background model image, $f_{j}(x, y, \mathrm{t})$ is the background subtraction image.

For moving objects detection is based on the computation of the threshold $\mathrm{X}$, if the background subtraction image is greater than $\mathrm{X}$, argues that the current frame is motion image, on the other hand, is considered to be static images. The selection of the threshold is the key to affect the moving object detection. Selection of the threshold $\mathrm{X}$ 
too small will cause a lot of noise, and too large will make moving object region fuzzy $[9,10]$.

The design in this paper is in light of the shortcomings of the existing algorithm, and applied to the indoor environment. So we select the background subtraction method and frame difference to detect intrusion objects. This design effectively improves the frame difference cavitations of the target objects and error of the light intensity difference.

The background subtraction method and frame difference have their own advantages and disadvantages. This paper combines these two methods, and makes use of advantages. This paper extracts the background frame and current frame image in the video image sequence, and computes background frame and current frame image difference to extract the complete target information, then extracts the current frame and the previous frame in the video image sequence, and computes current frame and previous frame difference to get the variation of the target. The twice differences make the intersection operation to get the fuzzy region image of moving target. It uses morphological filtering to processing target image to get the accurately complete area image of moving target.

The formula is

$$
\begin{gathered}
f_{z}(x, y, \mathrm{t})=\left\{\begin{array}{l}
0,\left|f_{m}(\mathrm{x}, \mathrm{y}, \mathrm{t})-f_{n}(\mathrm{x}, \mathrm{y}, \mathrm{t}-1)\right| T \\
1,\left|f_{m}(\mathrm{x}, \mathrm{y}, \mathrm{t})-f_{n}(\mathrm{x}, \mathrm{y}, \mathrm{t}-1)\right|>T
\end{array}\right. \\
f_{b}(x, y, \mathrm{t})=\left\{\begin{array}{l}
0,\left|f_{m}(\mathrm{x}, \mathrm{y}, \mathrm{t})-f_{n}(\mathrm{x}, \mathrm{y})\right| T \\
1,\left|f_{m}(\mathrm{x}, \mathrm{y}, \mathrm{t})-f_{n}(\mathrm{x}, \mathrm{y})\right|>T
\end{array}\right. \\
f_{s}(x, y, \mathrm{t})=f_{z}(x, y, \mathrm{t}) \& f d(x, y, \mathrm{t}) \\
z s(x, y, \mathrm{t})=\left\{\begin{array}{l}
0, \text { otherwise } \\
1, f_{s}(x, y, \mathrm{t})>1
\end{array}\right.
\end{gathered}
$$

Where $f z(x, y, \mathrm{t})$ is the current frame and the previous frame difference, $f b(x, y, \mathrm{t})$ is the current frame and background frame difference, $f b(x, y, \mathrm{t})$ is the edge contour of the objects detection, $T$ is the threshold. In the input video sequences of image, if the pixel $\mathrm{t}$ doesn't move, then the frame difference $d_{t}$ will obey the Gaussian distribution which means value $u$ is 0 , variance is $\delta^{2}$. The formula is

$$
F\left(d_{t}\right)=\frac{1}{\sqrt{2 \pi} \delta} e^{\frac{d t^{2}}{2 \delta^{2}}}
$$

According to the principle that appropriate threshold can eliminate a certain noise, to choose the threshold properly.

\section{Hardware Structure of the System}

This system is mainly to achieve the moving objects detection, alarm, sending mails and other functions. Its main modules are divided into five parts, the visual acquisition system, the visual detection system, data processing unit, the information feedback alarm system, the 
information communication system. Hardware structure diagram of the system is as shown in Figure 1.

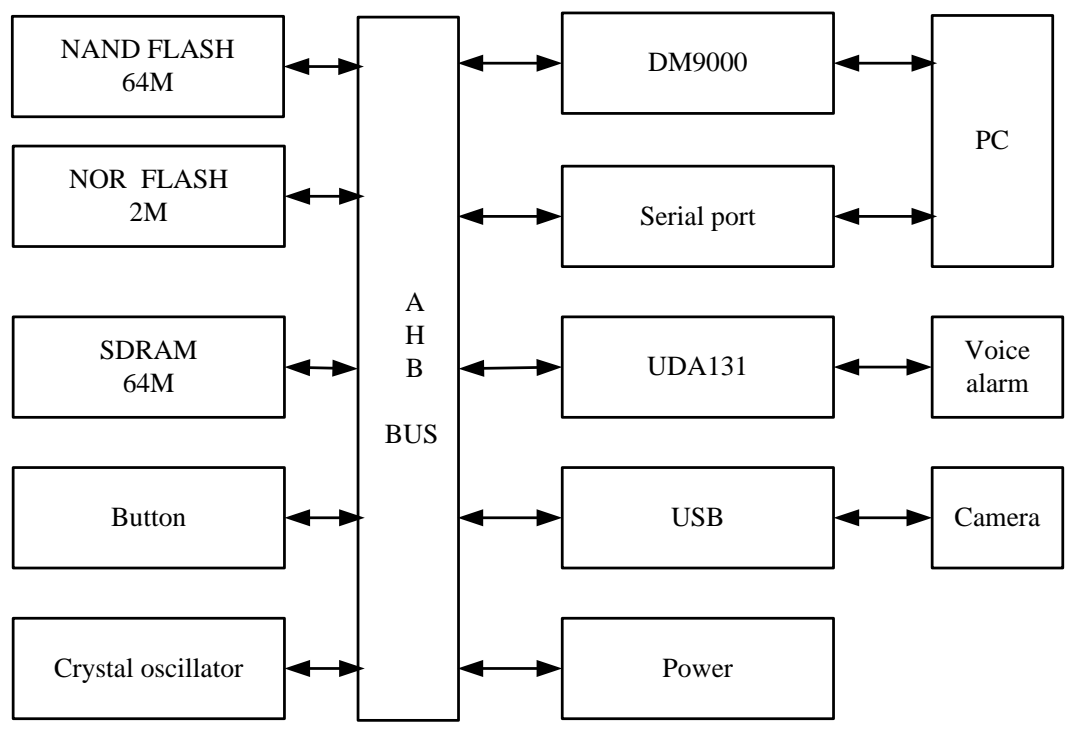

Figure 1. System Hardware Structure Diagram

The real-time video image acquisition system is completed by the video camera, the data processing system is completed by the processor with ARM as the core, to compute data and process instruction, and other functions. The visual detection system is completed by moving object detection program; this program combines background subtraction method and frame difference to detect the illegal invasion objects. Data processing unit is based on the processor with $\mathrm{S} 3 \mathrm{C} 2410$ as its core to process the data and instructions. The alarm system is completed by the alarm program, when someone presses the buttons, or illegal invasion objects are detected, the system will save the image and alarm. Mail system is completed by mail sending program, when the illegal invasion object is detected, system will send the image to the monitor automatically, to realize real-time monitoring. The information communication system is mainly completed by PC, including the system startup, turnoff, the observation of indoor environment, the invaders image query, etc.

\subsection{S3C2410 Peripheral Circuit Design}

3.1.1. USB External Circuit: USB is a external bus standard, used in specifying computer connections and communications with external devices, can be hot-plug and unified standards, reduces the amount of I/O interface, etc. Arm provides two HOST USB, a DEVICE USB, compatible with USB1.1 standard, supports both the low and high speed USB device. This design uses a USB connection device for data communications. Its interface circuit diagram is as shown in Figure 2. 

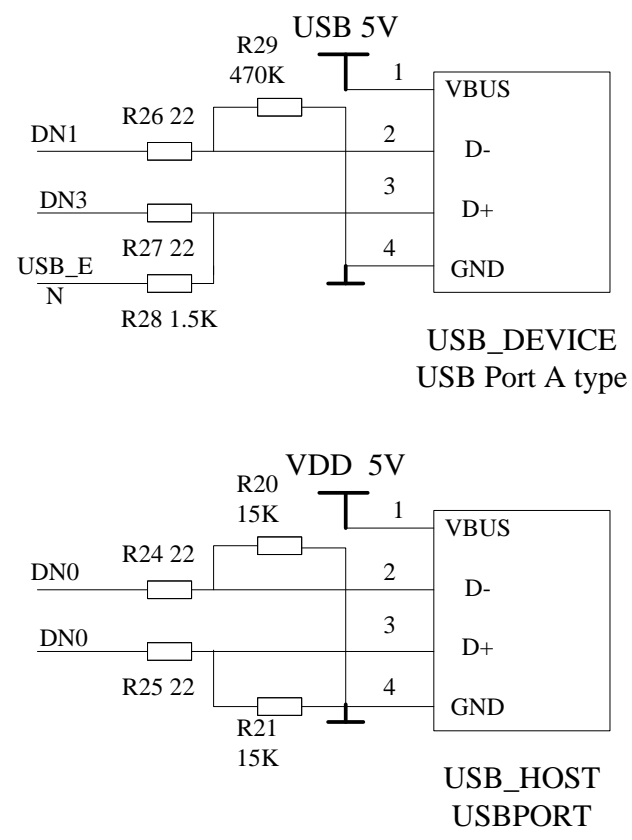

Figure 2. USB Circuit Diagram

3.1.2. Serial Interface Circuit: This design adopts the mode of serial communication, and sets data and instruction communication between computer and ARM. The serial interface circuit diagram is as shown in Figure 3.

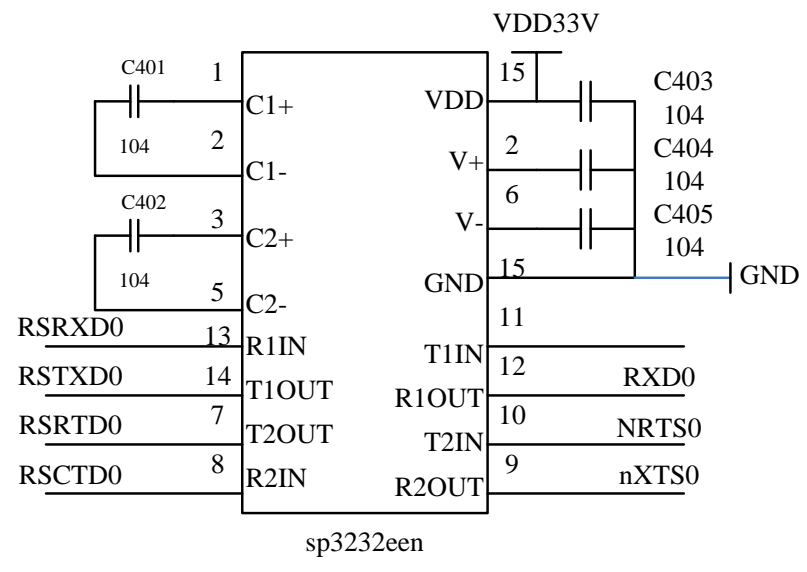

Figure 3. Serial Interface Circuit Diagram

3.1.3. Flash Memory Circuit: Flash memorizer is a kind of memory devices that is not volatile memory. The NOR and NAND are the two main technologies that are not volatile memory on the market currently. NAND flash memory storage unit uses the serial structure, reads and writes by page and block as the unit. Its advantage is that has large capacity and is suitable for mass storage. NOR flash memory is chip implementation. Its feature is high transmission efficiency, so it is suitable for reading program. The connection circuit is as shown in Figure 4. 


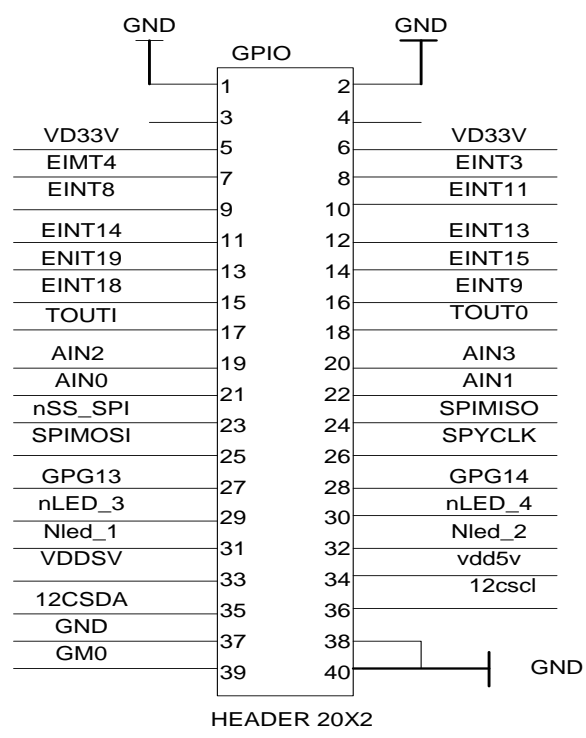

Figure 4. Flash Memory Circuit

\section{Design of each Function Module in System}

\subsection{Image Acquisition Part}

This design uses video cameras to collect images. V4L(video for Linux) is Linux standard for camera video. This standard defines a series of drivers, interface, and core applications programs. The USB video camera is its supported category. Video image acquisition process diagram is as shown in Figure 5.

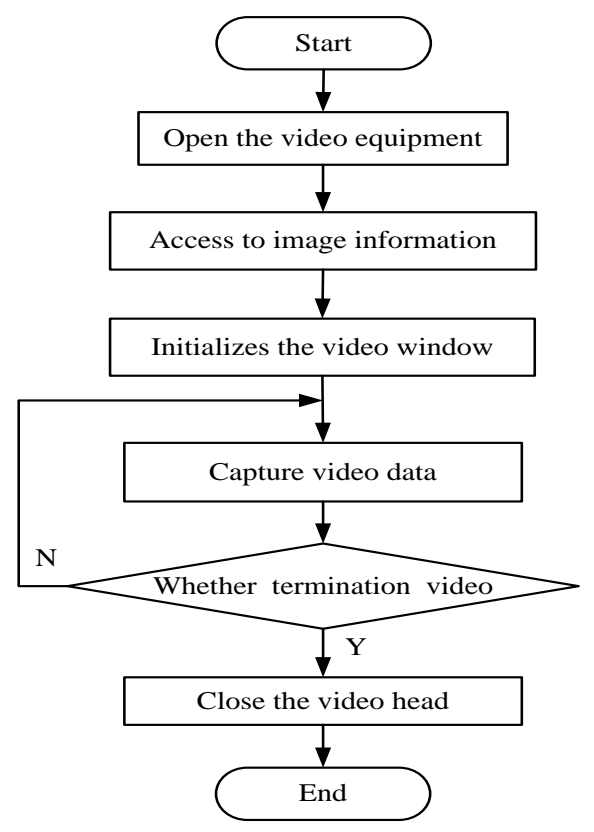

Figure 5. Flow chart of Video Image Acquisition 


\subsection{Alarm Program}

This design installs loudspeaker on the ARM, uses two ways to trigger the loudspeaker to produce alarm sound, warning the intruder to leave monitoring area. When testing program detects illegal invasion object indoor or someone pushes buttons artificially, alarm program starts executing, loudspeaker sounds alarm.

The alarm module uses MADALY player and voice alarm processing program. When illegal invasion object is detected in video image or someone pushes buttons artificially, program executes MADALY broadcast sound alarm function, at the same time, sends the mail to monitor preset mailbox inform monitor. The flow chart of alarm program is as shown in Figure6.

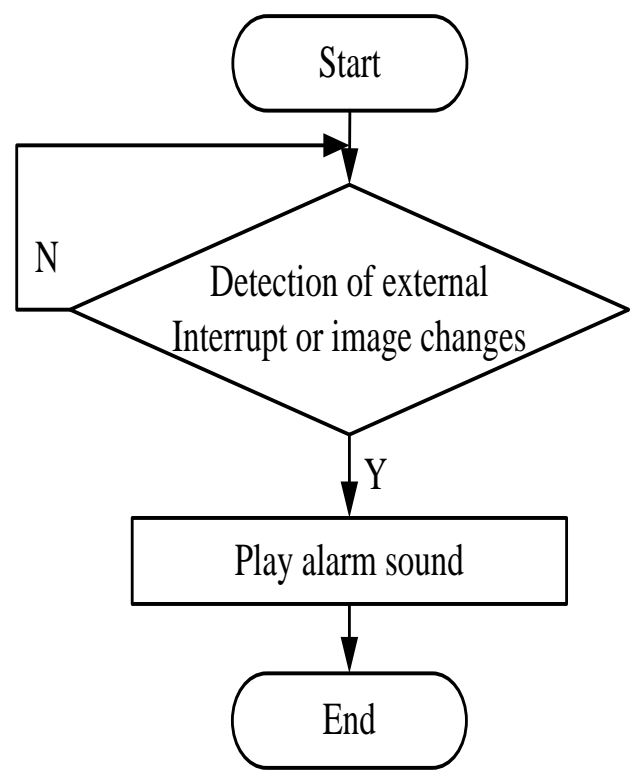

\section{Figure 6. Flow Chart of Alarm Program}

\subsection{Program of Sending Mail}

This design adds the mail-sending function in the embedded systems. When illegal objects invades, the system will save the pictures and send the pictures to the mailbox setted by monitor in advance. So monitor can timely know the case of illegal objects.

At present most of the mail needs to support SSL, this makes more difficult add the mailsending function in the embedded system. This design completes adding the mail-sending function in the embedded systems through the OPENSSL, SSMTP and MAILX.

MAILX is a relatively small mail sending and reading program. It supports for multiple SSL encryption mode and NSS, KERBEROS user authentication. In default situation, it will send mails to the local MTA (Mail Transfer Agent).

SMTP is supported by the TCP protocol, provides effective, reliable and simple mail transfer protocol, is a kind of mail service based on the TCP, establishes two-way transmission channel between letters and answers. Its biggest advantage is that can transmit across the network. The model of SMTP is as shown in Figure 7. 


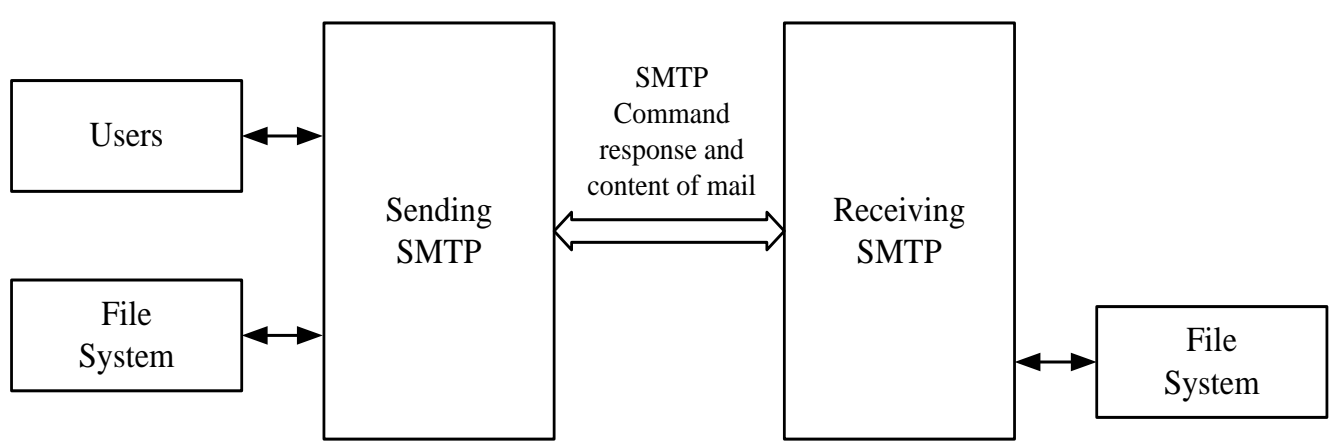

Figure 7. Model of SMTP

\section{Results of Design and Test}

This design is used in the base station monitoring of the telecommunication industry, the museum night monitoring, unmanned environment monitoring of living and working, and so on. So the design is simulated testing under the environment of laboratory. The experimental results show that the system can detect the moving object real-timely. The system detects the illegal objects into the monitoring system, and achieves stability about $0.6 \mathrm{~s}$ after strong change of light. Through a series of improved algorithm, this design makes the target detection accuracy and sensitivity improved. And the effect has obvious improved in practical application. After many experiments, we proved the reliability of this kind of motion detection algorithm. The test image is as shown in Figure 8.

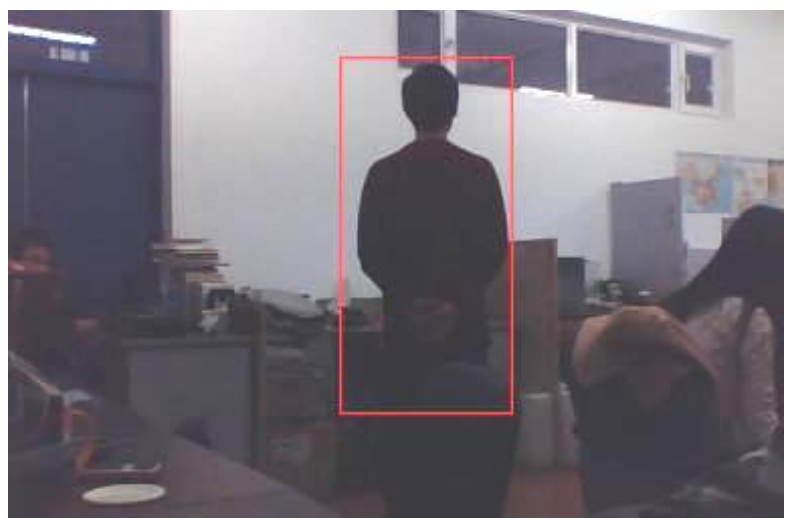

(a)

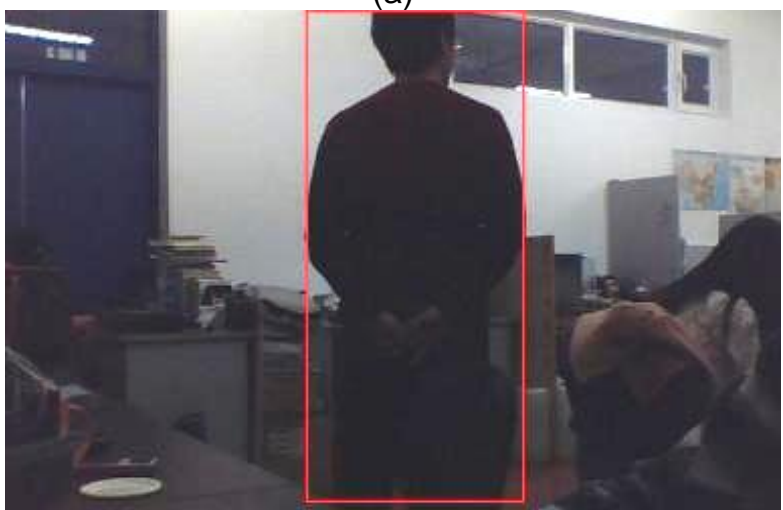

(b) 


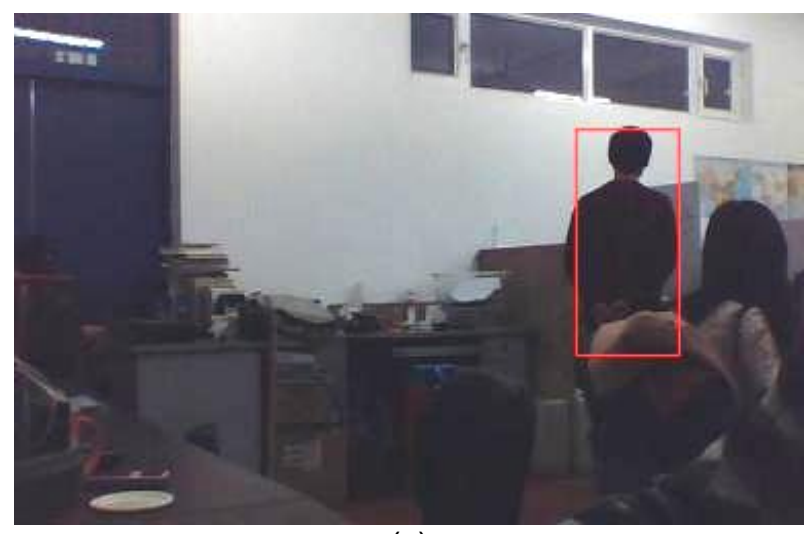

(c)

Figure 8. Experiment Environment Testing Images

\section{Conclusion}

This design is mainly studied the method of moving object detection in video sequences. This paper analyses the effects by speed of moving objects, light in environment and the algorithm complexity. Then this paper puts forward a kind of moving objects detection method base on the background subtraction method and the frame difference. This method uses the accuracy of background subtraction method and the strong light adaptability of frame difference. A large number of experiments show that this method has strong stability in the dynamic environment. At the same time, because of combining with the background subtraction method, the detection of moving objects has complete information, avoids the cavitation of moving target. This method is fast and stable, has very high practical value in video monitoring system.

\section{Acknowledgements}

This work was supported by grants from the Key project of science and technology in safety production's major production safety accident prevention (2012HLJ07) for funding this work.

\section{References}

[1] J. Bang, D. Kim and H. Eom, "Motion Object and Regional Detection Method Using Block-Based Background Difference Video Frames", IEEE International Conference, Seoul, Korea, (2012) August 19-22.

[2] S. Yanming and J. Kebin, "The Research and Implementation of Moving Object Detecting and Tracking in Intelligence Video Monitor System”, IEEE International Conference on Multimedia and Signal Processing, Guilin, China, (2011) May 14-15.

[3] L. Bing and S. Jianping, "Remote video monitoring system based on embedded linux and GPS", IEEE Conference on Computer Engineering and Technology (ICCET), Chengdu, China, (2010) April 16-18.

[4] W. Chongjing, Z. Xuzou and Y. Liu, Yuncai. Detecting Motion Patterns in Dynamic Crowd Scenes", IEEE International Conference, Hefei, China, (2011) August 12-15.

[5] L. Gang, Q. Shangbin, L. Ling and Z. Ruili, Chinese Journal of Scientific Instrument, vol. 8, no. 27, (2006).

[6] Li Feng, Yang Yanqiang. Modern Electronics Technique. 2, 36 (2013)

[7] Zhang Yong, Li Taijun, Li Meifang. Modern Electronics Technique. 8, 35 (2012)

[8] Yan Xiaoming. Journal of Putian University. 5, 18 (2011)

[9] Gao Kailiang, Qin Tuanfa, Wang Yizhi, Chang Kan. Telecommunication Engineering. 10, 51 (2011)

[10] Han Jun, Xiong Zhang, Gong shengrong, Sun Wenyan , Journal of Beijing University of Aeronautics and Astronautics. 4, 27 (2001) 
International Journal of Security and Its Applications Vol.7, No.6 (2013) 